Supporting Information

\title{
Experiments on the Capillary Condensation/Evaporation Hysteresis of Pure Fluids and Binary Mixtures in Cylindrical Nanopores
}

\author{
Xingdong Qiu ${ }^{a}$, Huan Yang ${ }^{a}$, Morteza Dejam ${ }^{a}$, Sugata P. Tan ${ }^{b}$, Hertanto Adidharma ${ }^{a, c^{*}}$ \\ ${ }^{a}$ Department of Petroleum Engineering, College of Engineering and Applied Science, University of Wyoming, 1000 \\ E. University Avenue, Laramie, WY 82071-2000, USA \\ ${ }^{b}$ Planetary Science Institute, Tucson, Arizona 85719-2395, USA \\ ${ }^{c}$ Department of Chemical Engineering, College of Engineering and Applied Science, University of Wyoming, 1000 \\ E. University Avenue, Laramie, WY 82071-2000, USA
}

${ }^{*}$ Corresponding author.

E-mail address: adidharm@uwyo.edu

Qiu et al. (2021) 


\section{Characterization of the porous materials}
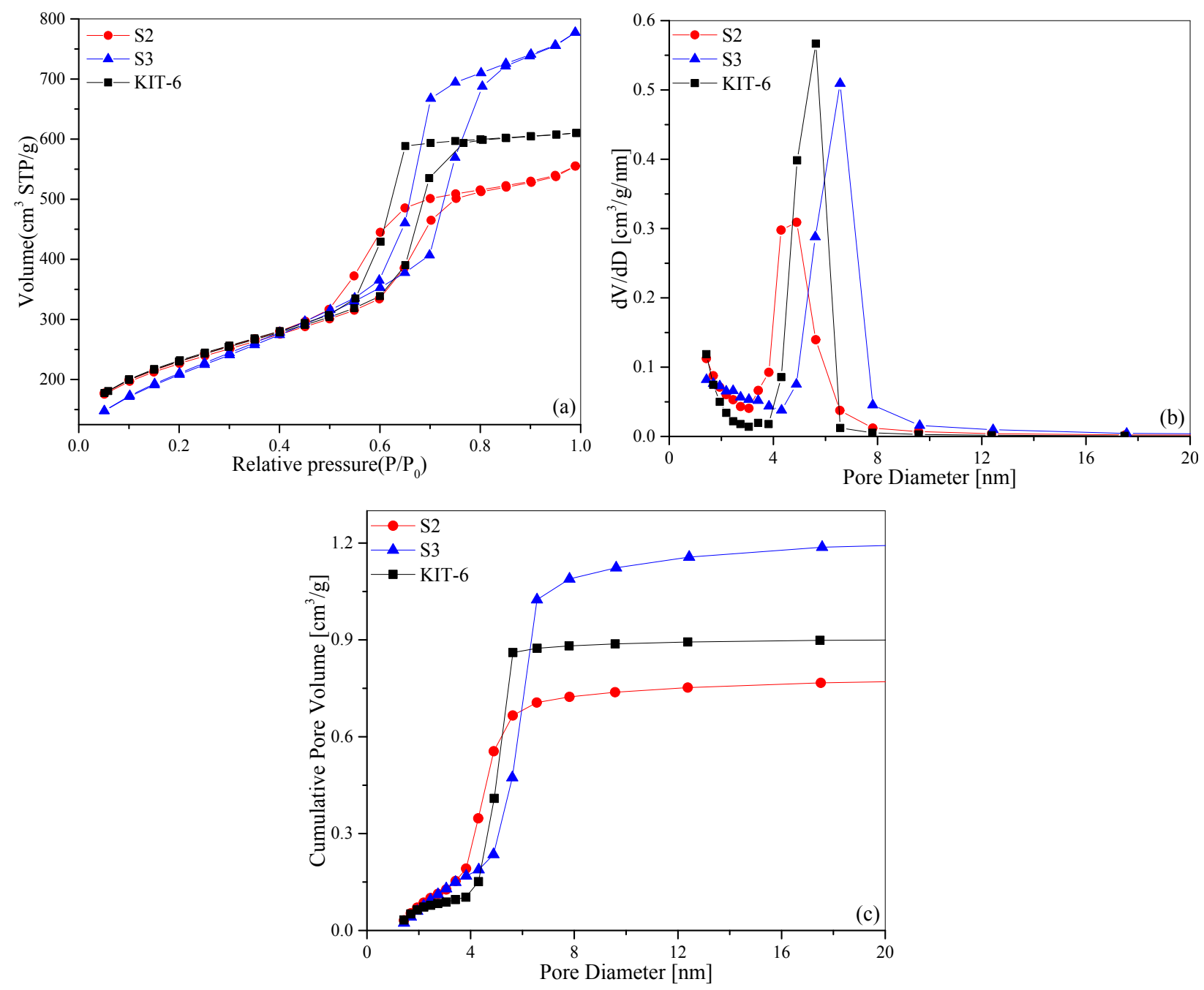

Figure S1. (a) Nitrogen adsorption-desorption isotherms, (b) Pore size distributions, and (c) Cumulative pore volume.

As shown in Figure S1 (a), the main physical properties, including BET surface area, total pore volume, as well as pore size distribution, of the three nanoporous samples used in this work has been characterized using the standard $\mathrm{N}_{2}$ adsorption isotherm measurement at $77 \mathrm{~K}$. The pore size is characterized by BJH and NLDFT by implementing a Generalized Adsorption Isotherm equation assuming that the total isotherm is the sum of a number of individual single-pore isotherms multiplied by their relative distribution over a range of pore sizes, from which the pore size distribution can be obtained. All of these are done using Quantachrome's data analysis software. As expected, all samples present micropores, that is pore size less than $2 \mathrm{~nm}$ according to IUPAC classification, ${ }^{1}$ as shown in Figure S1 (b). However, the pore diameter reported in Table 1 of the main manuscript corresponds to the peak of the pore size distribution of the mesopores, because as indicated in the main manuscript, those micropores are filled with 
gases at a much lower pressure than the capillary condensation and evaporation pressures within the mesopores. ${ }^{2,3}$ Consequently, the existence of micropores is considered not to have an appreciable impact on the first-order phase transition, i.e., capillary condensation/evaporation process occurring in the main mesopores. ${ }^{4,5}$ Note that compared to the pore volume of main mesopores, the pore volume of micropores is negligible in our case, as clearly shown in Figure S1 (c), the fact of which serve as the basis for our discussion in section 3.4.2 of the main manuscript.

\section{Experimental data measured in this work}

\begin{tabular}{|c|c|c|c|c|c|c|c|c|c|c|c|}
\hline \multicolumn{6}{|c|}{$\mathrm{CO}_{2} / \mathrm{S} 2$} & \multicolumn{6}{|c|}{$\mathrm{C}_{2} \mathrm{H}_{6} / \mathrm{S} 2$} \\
\hline $\mathrm{Tcc}[\mathrm{K}]$ & Pcc[bar] & $\mathrm{Hcc}[\mathrm{J}]$ & Tce $[\mathrm{K}]$ & Pce[bar] & Hce $[\mathrm{J}]$ & $\operatorname{Tcc}[\mathrm{K}]$ & Pcc[bar] & $\mathrm{Hcc}[\mathrm{J}]$ & Tce $[\mathrm{K}]$ & Pce[bar] & Hce $[\mathrm{J}]$ \\
\hline 238.49 & 9.57 & 0.3454 & 238.99 & 9.56 & 0.3476 & 237.08 & 6.54 & 0.3210 & 237.43 & 6.54 & 0.3241 \\
\hline 240.63 & 10.33 & 0.3305 & 241.05 & 10.33 & 0.3325 & 238.86 & 6.95 & 0.3057 & 239.15 & 6.96 & 0.3027 \\
\hline 241.93 & 10.84 & 0.3224 & 242.33 & 10.84 & 0.3208 & 241.16 & 7.51 & 0.2876 & 241.36 & 7.51 & 0.288 \\
\hline 244.53 & 11.90 & 0.3048 & 244.84 & 11.90 & 0.3018 & 243.00 & 7.98 & 0.2684 & 243.10 & 7.99 & 0.2657 \\
\hline 246.97 & 12.94 & 0.2863 & 247.20 & 12.94 & 0.2833 & 245.03 & 8.52 & 0.2574 & 245.05 & 8.53 & 0.2575 \\
\hline 250.04 & 14.37 & 0.2657 & 250.19 & 14.37 & 0.2667 & 247.64 & 9.26 & 0.2358 & 247.66 & 9.26 & 0.2369 \\
\hline 252.51 & 15.63 & 0.2543 & 252.61 & 15.63 & 0.2545 & 249.01 & 9.67 & 0.2252 & 249.05 & 9.67 & 0.2268 \\
\hline 254.80 & 16.83 & 0.2342 & 254.81 & 16.84 & 0.2321 & 252.45 & 10.75 & 0.2010 & 252.42 & 10.75 & 0.2021 \\
\hline 257.54 & 18.37 & 0.2181 & 257.57 & 18.38 & 0.2199 & 254.94 & 11.59 & 0.1870 & 254.96 & 11.60 & 0.1875 \\
\hline 259.85 & 19.74 & 0.2052 & 259.85 & 19.74 & & 256.95 & 12.30 & 0.1720 & 256.97 & 12.31 & 0.1731 \\
\hline 263.01 & 2178 & 0.1884 & 263.04 & 21.78 & 0.1861 & & & & & & \\
\hline \multicolumn{6}{|c|}{$\mathrm{CO}_{2} / \mathrm{KIT}-6$} & \multicolumn{6}{|c|}{$\mathrm{C}_{2} \mathrm{H}_{6} / \mathrm{KIT}-6$} \\
\hline $\mathrm{Tcc}[\mathrm{K}]$ & Pcc[bar] & $\mathrm{Hcc}[\mathrm{J}]$ & Tce $[\mathrm{K}]$ & Pce[bar] & Hce $[\mathrm{J}]$ & $\mathrm{Tcc}[\mathrm{K}]$ & Pcc[bar] & $\mathrm{Hcc}[\mathrm{J}]$ & Tce $[\mathrm{K}]$ & Pce[bar] & Hce $[\mathrm{J}]$ \\
\hline 242.26 & 11.12 & 0.3115 & 242.72 & 11.13 & 0.314 & 268.43 & 17.08 & 0.1064 & 268.43 & 17.07 & 0.105 \\
\hline 243.73 & 11.69 & 0.3070 & 244.13 & 11.68 & 0.3085 & 265.51 & 15.81 & 0.1209 & 265.48 & 15.80 & 0.1218 \\
\hline 245.70 & 12.54 & 0.2965 & 246.06 & 12.54 & 0.2947 & 263.03 & & 0.1371 & 263.03 & 14.78 & 0.1356 \\
\hline 247.47 & 13.29 & 0.2836 & 247.77 & 13.30 & 0.2853 & 260.84 & & 0.1509 & 260.85 & 13.93 & 0.1511 \\
\hline 250.25 & 14.61 & 0.2675 & 250.51 & 14.60 & 0.2672 & 258.70 & 13.12 & 0.1608 & 258.73 & 13.12 & 0.1602 \\
\hline 253.82 & 16.41 & 0.2520 & 253.97 & 16.41 & 0.2515 & 257.06 & 12.50 & 0.1719 & 257.06 & 12.50 & 0.1716 \\
\hline 256.75 & 18.03 & 0.2357 & 256.86 & 18.04 & & 253.87 & & 0.1895 & 253.90 & 11.41 & 0.1903 \\
\hline 259.39 & 19.56 & 0.2203 & 259.36 & & 0.2218 & & & 0.2065 & 251.31 & 10.57 & 0.205 \\
\hline 261.26 & 20.73 & 0.2139 & 261.26 & 20.74 & 0.2141 & 248.92 & 9.83 & 0.2223 & 248.94 & 9.84 & 0.2241 \\
\hline 263.68 & 22.32 & 0.2014 & 263.65 & 22.31 & 0.1992 & 246.74 & 9.21 & 0.2361 & 246.78 & 9.21 & 0.2359 \\
\hline 267.42 & 24.93 & 0.1850 & & & & 244.49 & 8.58 & 0.2501 & 244.62 & 8.59 & 0.2503 \\
\hline 271.55 & 28.07 & 0.1620 & 271.57 & 28.07 & 0.1638 & 241.62 & 7.84 & 0.2686 & 241.85 & 7.85 & 0.2698 \\
\hline \multicolumn{6}{|c|}{$\mathrm{CO}_{2} / \mathrm{S} 3$} & \multicolumn{6}{|c|}{$\mathrm{C}_{2} \mathrm{H}_{6} / \mathrm{S} 3$} \\
\hline $\mathrm{Tcc}[\mathrm{K}]$ & Pcc[bar] & $\mathrm{Hcc}[\mathrm{J}]$ & Tce $[\mathrm{K}]$ & Pce[bar] & Hce $[\mathrm{J}]$ & $\mathrm{Tcc}[\mathrm{K}]$ & Pcc[bar] & $\mathrm{Hcc}[\mathrm{J}]$ & Tce $[\mathrm{K}]$ & Pce[bar] & Hce $[\mathrm{J}]$ \\
\hline 243.41 & 12.18 & 0.2406 & 244.31 & 12.20 & 0.2412 & 241.74 & 8.17 & 0.2665 & 242.65 & 8.20 & 0.2677 \\
\hline 245.88 & 13.26 & 0.2286 & 246.74 & 13.28 & 0.2306 & 245.09 & 9.09 & 0.2491 & 245.88 & 9.11 & 0.2478 \\
\hline 248.85 & 14.65 & 0.2192 & 249.63 & 14.67 & 0.2198 & 248.33 & 10.06 & 0.2323 & 249.02 & 10.09 & 0.2326 \\
\hline 251.27 & 15.92 & 0.2084 & 251.97 & 15.92 & 0.2091 & 250.79 & 10.82 & 0.2191 & 251.34 & 10.84 & 0.2152 \\
\hline 253.99 & 17.32 & 0.1988 & 254.62 & 17.33 & 0.1984 & 253.23 & 11.65 & 0.2075 & 253.57 & 11.67 & 0.2085 \\
\hline 256.22 & 18.60 & 0.1865 & 256.74 & 18.61 & 0.1857 & 256.23 & 12.69 & 0.1922 & 256.34 & 12.69 & 0.1913 \\
\hline
\end{tabular}




\begin{tabular}{|c|c|c|c|c|c|c|c|c|c|c|c|}
\hline 258.82 & 20.19 & 0.1807 & 259.28 & 20.18 & 0.1801 & 258.11 & 13.40 & 0.1843 & 258.19 & 13.40 & 0.1831 \\
\hline 261.18 & 21.65 & 0.1711 & 261.50 & 21.66 & 0.1716 & 260.67 & 14.40 & 0.1731 & 260.68 & 14.41 & 0.1756 \\
\hline 264.11 & 23.61 & 0.1616 & 264.32 & 23.61 & 0.1639 & 262.67 & 15.22 & 0.1624 & 262.70 & 15.26 & 0.1611 \\
\hline 266.90 & 25.64 & 0.1468 & 266.97 & 25.63 & 0.1475 & 265.01 & 16.22 & 0.1556 & 264.98 & 16.23 & 0.1567 \\
\hline 269.53 & 27.65 & 0.1364 & 269.57 & 27.65 & 0.1364 & 268.41 & 17.79 & 0.1396 & 268.42 & 17.79 & 0.1397 \\
\hline 273.06 & 30.48 & 0.1282 & 273.05 & 30.48 & 0.1284 & 273.52 & 20.27 & 0.1160 & 273.50 & 20.28 & 0.1138 \\
\hline 276.46 & 33.44 & 0.1172 & 276.47 & 33.44 & 0.1155 & & & & & & \\
\hline \multicolumn{6}{|c|}{$12 \% \mathrm{CH}_{4} / 88 \% \mathrm{CO}_{2} / \mathrm{S} 3$} & \multicolumn{6}{|c|}{$15 \% \mathrm{CH}_{4} / 85 \% \mathrm{C}_{2} \mathrm{H}_{6} / \mathrm{S} 3$} \\
\hline $\operatorname{Tcc}[\mathrm{K}]$ & Pcc[bar] & $\mathrm{Hcc}[\mathrm{J}]$ & Tce $[\mathrm{K}]$ & Pce[bar] & Hce $[\mathrm{J}]$ & $\operatorname{Tcc}[\mathrm{K}]$ & Pcc[bar] & $\mathrm{Hcc}[\mathrm{J}]$ & Tce $[\mathrm{K}]$ & Pce[bar] & Hce $[\mathrm{J}]$ \\
\hline 240.08 & 12.64 & 0.0657 & 241.26 & 12.64 & 0.066 & 276.99 & 27.54 & - & 277.02 & 27.55 & - \\
\hline 242.17 & 13.62 & 0.0630 & 243.21 & 13.61 & 0.0625 & 272.91 & 24.68 & 0.0409 & 272.93 & 24.69 & 0.0399 \\
\hline 244.32 & 14.73 & 0.0602 & 245.35 & 14.73 & 0.061 & 269.4 & 22.43 & 0.0485 & 269.37 & 22.45 & 0.0454 \\
\hline 248.31 & 16.88 & 0.0530 & 249.35 & 16.88 & 0.054 & 265.47 & 20.15 & 0.0489 & 265.5 & 20.15 & 0.0499 \\
\hline 250.32 & 18.08 & 0.0505 & 251.13 & 18.09 & 0.0521 & 261.95 & 18.23 & 0.0575 & 262.02 & 18.23 & 0.0562 \\
\hline 254.16 & 20.49 & 0.0463 & 254.9 & 20.5 & 0.0458 & 260.22 & 17.34 & 0.0606 & 260.32 & 17.33 & 0.0609 \\
\hline 256.52 & 22.11 & 0.0415 & 257.1 & 22.12 & 0.042 & 257.18 & 15.84 & 0.0652 & 257.31 & 15.85 & 0.0668 \\
\hline 260.13 & 24.8 & 0.0360 & 260.6 & 24.8 & 0.0355 & 253.47 & 14.21 & 0.0709 & 253.79 & 14.22 & 0.0718 \\
\hline 262.9 & 27.1 & 0.0343 & 263.35 & 27.11 & 0.033 & 250.17 & 12.83 & 0.0762 & 250.57 & 12.83 & 0.0758 \\
\hline 265 & 28.86 & 0.0315 & 265.3 & 28.85 & 0.0301 & 247.25 & 11.73 & 0.0817 & 247.74 & 11.73 & 0.0811 \\
\hline 267.55 & 31.17 & 0.0278 & 267.82 & 31.19 & 0.028 & 243.29 & 10.35 & 0.0876 & 243.93 & 10.36 & 0.0865 \\
\hline 269.81 & 33.32 & 0.0235 & 269.96 & 33.32 & 0.0237 & 239.88 & 9.26 & 0.0957 & 240.66 & 9.26 & 0.097 \\
\hline 271.85 & 35.43 & - & 271.88 & 35.43 & - & 236.03 & 8.14 & 0.1042 & 236.93 & 8.16 & 0.103 \\
\hline 274.81 & 38.56 & 0.0188 & 274.84 & 38.57 & 0.0192 & & & & & & \\
\hline 278.13 & 42.44 & - & 278.15 & 42.47 & - & & & & & & \\
\hline
\end{tabular}

\section{Determination of $T_{h e}$ from curve fitting}

From Figure 7 of the main manuscript, we can get the error of $\left(P_{h e} / T_{h e}\right)$ from the linear regression of the irreversible part:

$$
\frac{P_{c c}}{T_{c c}}=A+B\left(T_{c e}-T_{c c}\right) \quad \text { with } A \pm \varepsilon_{A} \text { and } B \pm \varepsilon_{B}
$$

At $T_{h e}$, the second term vanishes, and therefore:

$$
\frac{P_{h e}}{T_{h e}}=A \pm \varepsilon_{A}
$$

The condensation curves in Figure 6 of the main manuscript can be fitted to the Clapeyron equation ${ }^{6,7}$ through another linear regression:

$$
\ln P_{c c}=C+\frac{D}{T_{c c}} \quad \text { with } C \pm \varepsilon_{C} \text { and } D \pm \varepsilon_{D}
$$

Subtract both side with $\ln T_{c c}$ :

$$
\ln \frac{P_{c c}}{T_{c c}}=C+\frac{D}{T_{c c}}-\ln T_{c c}
$$

Written completely with errors at $T_{h e}$ :

$$
\ln \left(A \pm \varepsilon_{A}\right)=\left(C \pm \varepsilon_{C}\right)+\frac{\left(D \pm \varepsilon_{D}\right)}{T_{c h}}-\ln T_{h e}
$$

Qiu et al. (2021) 
$T_{h e}$ can be calculated from this nonlinear Eq (S-5) without including the errors. However, to find the error of $T_{h e}, T_{h e}$ must be solved from this equation with all combination of the signs of the errors $\left( \pm \varepsilon_{A}\right.$, $\pm \varepsilon_{C}$, and $\pm \varepsilon_{D}$ ) to find the maximum and minimum values of $T_{h e}$, so that we will have the upper error and lower error of $T_{h e}$ :

$$
\begin{gathered}
\varepsilon_{U}=T_{h e}^{\max }-T_{h e} \\
\varepsilon_{L}=T_{h e}-T_{h e}^{\min }
\end{gathered}
$$

Finally, the $T_{h e}$ is expressed with an error, which turns out to be nearly symmetric for our cases here:

$$
\left(T_{h e}\right)_{-\varepsilon_{L}}^{+\varepsilon_{U}} \approx T_{h e} \pm \varepsilon
$$

The complete calculation results are shown in Table S1 below.

Table $\mathrm{S} 1$. The linear regressions and the resulting $T_{h e}$

\begin{tabular}{c|cccc}
\hline System & $\mathrm{A} \pm \varepsilon_{A}$ & $\mathrm{C} \pm \varepsilon_{C}$ & $\mathrm{D} \pm \varepsilon_{D}$ & $T_{h e} \pm \varepsilon[\mathrm{K}]$ \\
\hline $\mathrm{CO}_{2}-\mathrm{S} 2$ & $0.0659 \pm 0.00198$ & $11.08656 \pm 0.00674$ & $-2105.5754 \pm 1.68206$ & $254.7 \pm 1.5$ \\
\hline $\mathrm{C}_{2} \mathrm{H}_{6}-\mathrm{S} 2$ & $0.0352 \pm 7.36289 \mathrm{E}-4$ & $10.04748 \pm 0.00536$ & $-1936.8826 \pm 1.31973$ & $245.5 \pm 1.2$ \\
\hline $\mathrm{CO}_{2}-\mathrm{KIT} 6$ & $0.07703 \pm 0.00323$ & $10.99841 \pm 0.00651$ & $-2081.27082 \pm 1.6583$ & $260.1 \pm 2.0$ \\
\hline $\mathrm{C}_{2} \mathrm{H}_{6}-\mathrm{KIT} 6$ & $0.03839 \pm 0.00121$ & $9.86414 \pm 0.00626$ & $-1886.15504 \pm 1.59417$ & $247.8 \pm 1.7$ \\
\hline $\mathrm{CO}_{2}-\mathrm{S} 3$ & $0.10095 \pm 0.00157$ & $10.94484 \pm 0.00775$ & $-2055.36627 \pm 2.00561$ & $268.9 \pm 1.2$ \\
\hline $\mathrm{C}_{2} \mathrm{H}_{6}-\mathrm{S} 3$ & $0.05305 \pm 7.70822 \mathrm{E}-4$ & $9.92156 \pm 0.00684$ & $-1890.79884 \pm 1.75332$ & $259.0 \pm 1.2$ \\
\hline $\mathrm{CH}_{4} \mathrm{CO}_{2}-\mathrm{S} 3$ & $0.13334 \pm 0.00189$ & $11.36828 \pm 0.01562$ & $-2121.07674 \pm 4.034$ & $272.8 \pm 1.8$ \\
\hline $\mathrm{CH}_{4} \mathrm{C}_{2} \mathrm{H}_{6}-\mathrm{S} 3$ & $0.07071 \pm 0.00107$ & $10.32556 \pm 0.02095$ & $-1943.6785 \pm 5.35544$ & $262.5 \pm 2.3$ \\
\hline
\end{tabular}

\section{Lorentzian shape of condensation exothermic peak and evaporation endothermic peak}

In Figures 4 and 5 of the main manuscript, when the temperatures are in their respective reversible region, the shapes of peaks of both capillary condensation and evaporation are symmetric after the inclined baselines are accounted for. However, as discussed in the main manuscript, only the shape of exothermic peak of capillary condensation remains symmetric while that of the evaporation peak distorts in the irreversible region. The symmetric shape obeys Lorentzian distribution, as typically shown in Figure S2 below for both exothermic and endothermic peaks in the reversible region.

In Figure S2, the left-column is for confined pure fluid and the right-hand column is for confined mixture. After subtracting the inclined baselines in thermograms shown in the top row, the peaks are fitted to Lorentzian. The fitting results are almost perfect. Furthermore, the fitted parameters confirm that the 
condensation and the corresponding evaporation occur at the same temperature, which is the peak of the respective Lorentzian, i.e., $269.5 \mathrm{~K}$ for the pure fluid and $265.5 \mathrm{~K}$ for the mixture.
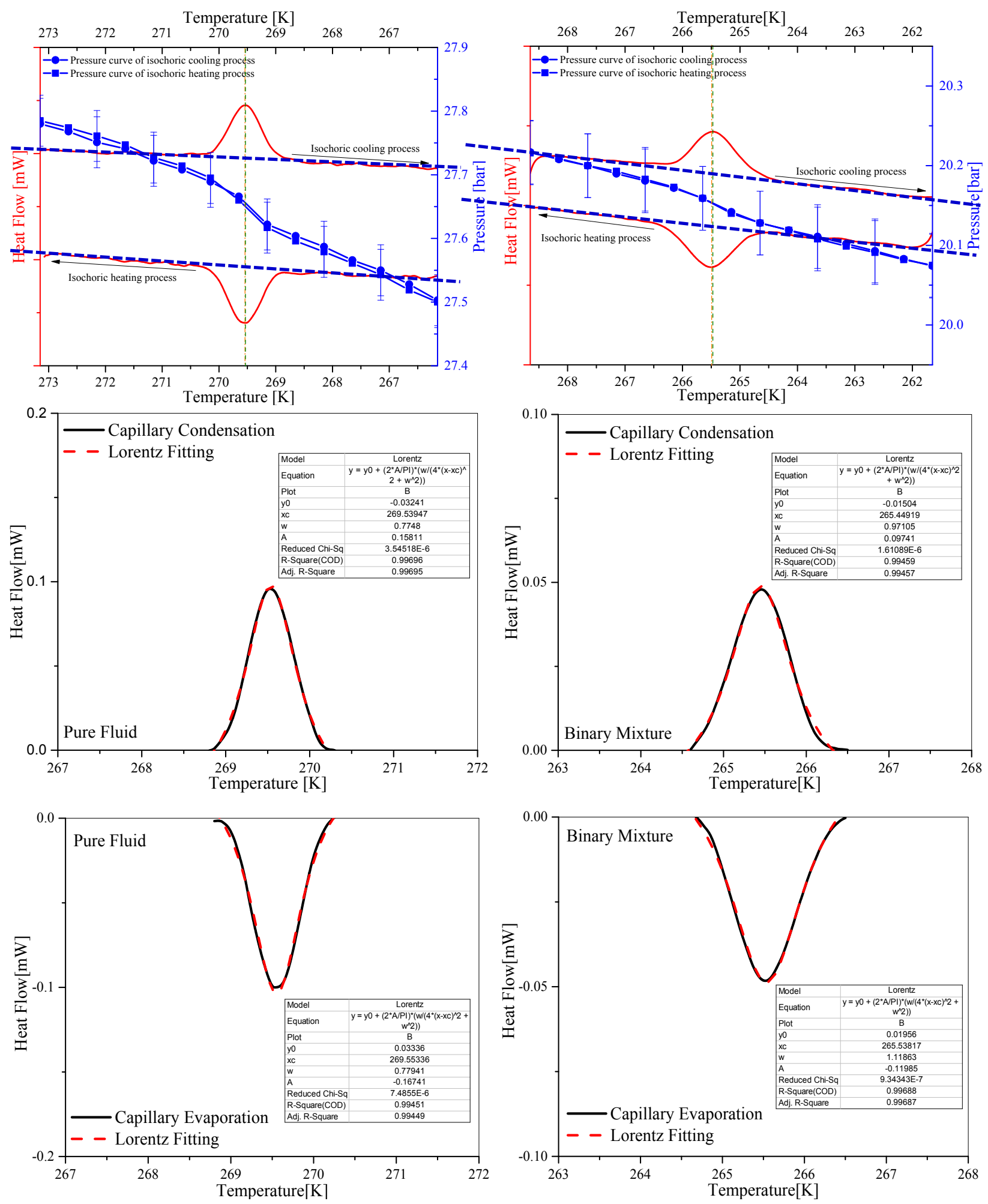

Qiu et al. (2021) 
Figure S2. Lorentzian fitting to typical exothermic and endothermic peaks in the reversible region for pure fluid (left-hand column) and mixture (right-hand column) in nanopores after the baselines (blue broken lines in the top row) are subtracted.

For interested readers, the Lorentzian shape here can be explained to arise due to the similar physics that occurs in optical spectroscopy ${ }^{8-10}$ The broadening of a spectral line happens because the light emission is perturbed by the presence of gas (at a pressure and density). Note that the light emission or absorption is analogous to heat release or adsorbed at condensation or evaporation on the thermogram, as all are forms of energy. However, the perturbing agent is different. In spectroscopy it is the gas, but in confined phase transition, it is the porous medium.

Physically, Lorentzian distribution can happen due to the perturbation that introduces noise $N:{ }^{11}$

$$
d H / d T=F(H, T)+N(T)
$$

On thermograms, we quickly recognize that $d H / d T$ represents the heat flow, such as shown in Figure S2, $H$ is the enthalpy, $T$ is the temperature, $F(H, T)$ is a function of the heat involved in phase transition (thus a function of $H^{\mathrm{V}}-H^{\mathrm{L}}$ between confined vapor and liquid), and $N(T)$ is the noise due to the pore size distribution (PSD) of the nanopores. It is this noise that introduces the broadening. Ideally, without $N(T)$ at capillary condensation, the profile of thermogram is $F(H, T)=\left(H^{\mathrm{V}}-H^{\mathrm{L}}\right) \delta\left(T-T_{c c}\right)$, where $\delta\left(T-T_{c c}\right)$ is the delta function at $T=T_{c c}$ (capillary-condensation temperature).

Eq (S-8) can immediately be applied to the derivative of an adsorption isotherm in adsorption/desorption experiments, such as those in Barsotti et al. ${ }^{12}$ with the following changes in variables:

$$
d V / d P=F(V, P)+N(P)
$$

where $V$ is the adsorbed amount of confined fluid, $P$ is pressure, $F(V, P)$ is a function of the adsorbed amount involved in phase transition (thus a function of $V^{\mathrm{L}}-V^{\mathrm{V}}$ between confined vapor and liquid), and $N(P)$ is the noise due to the PSD of the nanopores. Ideally, without $N(P)$ at capillary condensation, $F(V, P)=\left(V^{\mathrm{L}}-V^{\mathrm{V}}\right)$ $\delta\left(P-P_{c c}\right)$, where $\delta\left(P-P_{c c}\right)$ is the delta function at $P=P_{c c}$ (capillary-condensation pressure). Obviously, the analogy to the thermogram peaks is exact.

\section{Density of confined liquid-like phase at condensation and evaporation}

The Clayperon Equation is shown to be applicable to both capillary condensation and evaporation in the irreversible region sufficiently below $T_{h e}{ }^{2,13}$ even though the linearity of the evaporation branch is still uncertain near $T_{h e}$. Accordingly, ${ }^{6,7}$ we have

$$
\begin{aligned}
& \ln P_{c c}=-\frac{L_{c c} / R I}{\Delta Z_{c c} T_{c c}}+\text { constant } \\
& \ln P_{c e}=-\frac{L_{c e} / R I}{\Delta Z_{c e} T_{c e}}+\text { constant }
\end{aligned}
$$


where $L_{c c}$ and $L_{c e}$ are the molar heat of capillary condensation and capillary evaporation, respectively; $\Delta Z_{c c}$ and $\Delta Z_{c e}$ are the compressibility factor difference of the equilibrium phases during capillary condensation and capillary evaporation, respectively; $R$ is the gas constant.
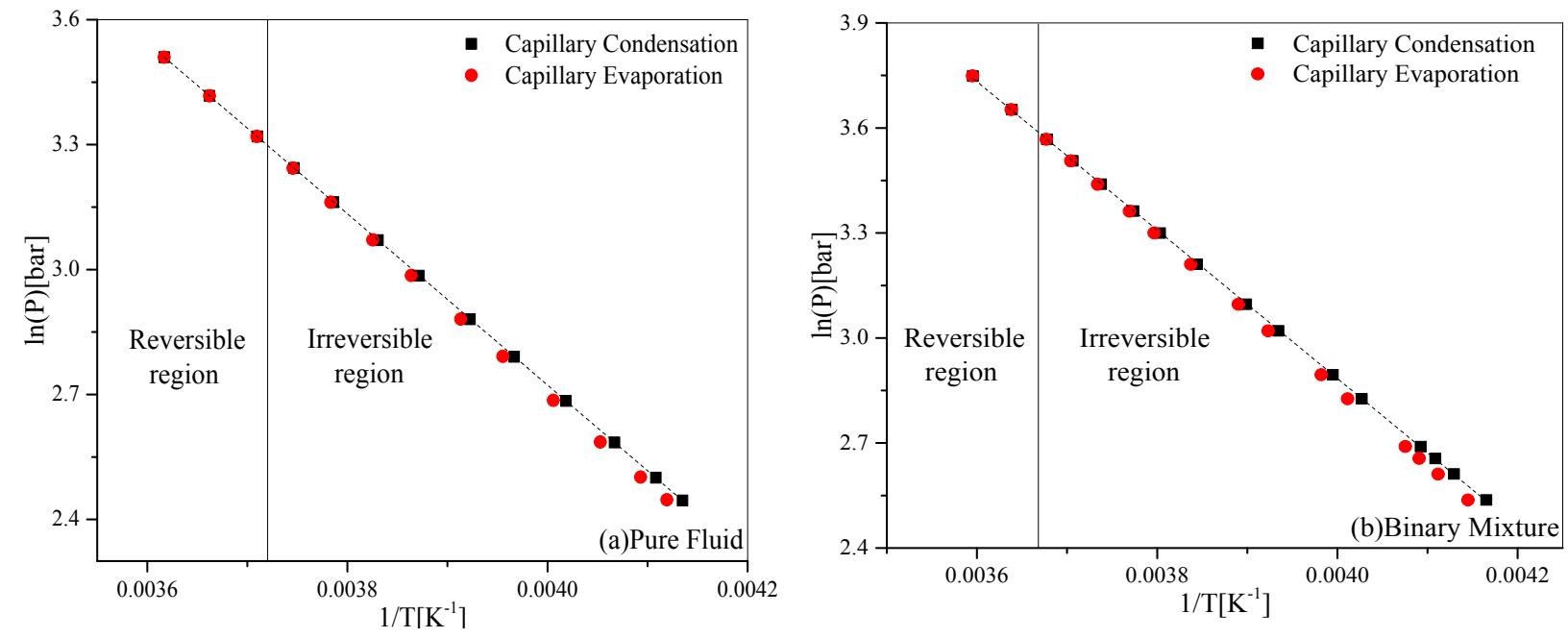

Figure S3. Validity of Clapeyron equation $(\ln P$ vs. $1 / T)$ for capillary condensation and capillary evaporation of (a) pure fluid $\left(\mathrm{CO}_{2} / \mathrm{S} 3\right)$ and (b) binary mixture $\left(\mathrm{CH}_{4} / \mathrm{CO}_{2} / \mathrm{S} 3\right)$. The dashed line is linear regression of capillary condensation.

As shown in Figure S3, in the irreversible region, the slope of the linear equation of capillary condensation is larger than that of capillary evaporation, thus we have

$$
-\frac{L_{c c}}{\Delta Z_{c c}}>-\frac{L_{c e}}{\Delta Z_{c e}}
$$

so that,

$$
\frac{L_{c e}}{\Delta Z_{c e}}>\frac{L_{c c}}{\Delta Z_{c c}}
$$

Based on the conservation of mass, the amount of liquid-like phase just after capillary condensation should be the same as that just before capillary evaporation. As indicated in Figure 9 of the main manuscript, we also find that the total heat of capillary condensation is identical to that of capillary evaporation for all examined systems, even when the hysteresis is present. Consequently,

$$
L_{c e}=L_{c c}
$$

Then we have,

$$
\Delta Z_{c c}>\Delta Z_{c e}
$$

Accordingly, ${ }^{14}$ the full expressions of $\Delta Z_{c c}$ and $\Delta Z_{c e}$ for confined systems can be written as

$$
\begin{aligned}
& \Delta Z_{c c}=Z^{V_{c c}}-Z^{l_{c c}} P^{V_{c c}} / P^{l_{c c}} \\
& \Delta Z_{c e}=Z^{V_{c e}}-Z^{l_{c e}} P^{V_{c e}} / P^{l_{c e}}
\end{aligned}
$$


where $Z^{V_{c c}}$ and $Z^{V_{c e}}$ are the compressibility factors of vapor phase at capillary condensation and evaporation, respectively. As indicated in the main manuscript, for vapor phase in bulk, $P / T$ that corresponds to density is effectively unchanged at capillary condensation and evaporation as any non-idealities of the isochoric process are negligible at both conditions. In this case, $Z$ is expected insensitive to a small difference between capillary condensation and evaporation. In other words, we have $Z^{V_{c c}}=Z^{V_{c e}} . Z^{l_{c c}}$ and $Z^{l_{c e}}$ are compressibility factors of liquid-like phase in nanopores at capillary condensation and evaporation, respectively; $P^{V_{c c}}, P^{l_{c c}}, P^{V_{c e}}$ and $P^{l_{c e}}$ are pressures of vapor phase and liquid-like phase at capillary condensation and evaporation, respectively. Therefore, we have

$$
Z^{l_{c e}} P^{V_{c e}} / P^{l_{c e}}>Z^{l_{c c}} P^{V_{c c}} / P^{l_{c c}}
$$

By definition,

$$
\begin{aligned}
& Z^{l_{c c}}=\frac{P^{l_{c c}}}{\rho^{l^{c c}} R T^{c c}} \\
& Z^{l_{c e}}=\frac{P^{l_{c e}}}{\rho^{l^{c e}} R T^{c e}}
\end{aligned}
$$

so that,

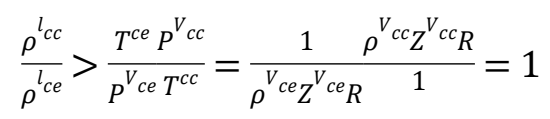

Finally, we have,

$$
\rho^{l_{c c}}>\rho^{l_{c e}}
$$

In other words, based on the equality of the total heat of capillary condensation and evaporation, it can be further concluded that the density of confined liquid-like phase at evaporation is less than that at condensation, which can serve as the evidence that supports the contraction of the pore wall by negative pressure (tension) at condensation, while relaxation somewhat at a higher temperature at evaporation. ${ }^{15}$ 


\section{References}

1. Thommes, M.; Kaneko, K.; Neimark, A. V.; Olivier, J. P.; Rodriguez-Reinoso, F.; Rouquerol, J.; Sing, K. S. W. Physisorption of gases, with special reference to the evaluation of surface area and pore size distribution (IUPAC Technical Report). Pure Appl. Chem., 2015, 87, 1051-1069.

2. Morishige, K.; Ito, M. Capillary condensation of nitrogen in MCM-41 and SBA-15. J. Chem. Phys., 2002, 117, 8036-8041.

3. Morishige, K.; Tarui, N. Capillary Condensation of Nitrogen in Ordered Mesoporous Silica with Bicontinuous Gyroid Structure. J. Phys. Chem. C, 2007, 111, 280-285.

4. Grosman, A.; Ortega, C. Nature of Capillary Condensation and Evaporation Processes in Ordered Porous Materials. Langmuir, 2005, 21, 10515-10521.

5. Kleitz, F.; Berube, F.; Guillet-Nicolas, R.; Yang, C. M.; Thommes, M. Probing Adsorption, Pore Condensation, and Hysteresis Behavior of Pure Fluids in Three-Dimensional Cubic Mesoporous KIT-6 Silica. J. Phys. Chem. C, 2010, 114, 9344-9355.

6. Tan, S. P.; Qiu, X.; Dejam, M.; Adidharma, H. Critical Point of Fluid Confined in Nanopores: Experimental Detection and Measurement. J. Phys. Chem. C, 2019, 123, 9824-9830.

7. Qiu, X.; Tan, S. P.; Dejam, M.; Adidharma, H. Experimental Study on the Criticality of a Methane/Ethane Mixture Confined in Nanoporous Media. Langmuir, 2019, 35, 11635-11642.

8. Svelto, O. Principles of Lasers, 5th edition, Springer, New York, 2010, p.46.

9. Fukuchi, T.; Shiina, T. Industrial Applications of Laser Remote Sensing, Bentham Science Publishers, 2012, Ch.3, p.40.

10. Peach, G. Theory of the pressure broadening and shift of spectral lines, Adv. Phys., 1981, 30, 367-474. 11. Koverda, V. P.; Skokov, V. N.; Skripov, V. P. 1/f noise in a nonequilibrium phase transition: experiment and mathematical model. J. Exp. Theor. Phys., 1998, 86, 953-958.

12. Barsotti, E.; Tan, S. P.; Piri, M.; Chen, J. H. Phenomenological Study of Confined Criticality: Insights from the Capillary Condensation of Propane, n-Butane, and n-Pentane in Nanopores. Langmuir, 2018, 34, 4473-4483.

13. Morishige, K.; Nakamura, Y. Nature of Adsorption and Desorption Branches in Cylindrical Pores. Langmuir, 2004, 20, 4503-4506.

14. Tan, S. P.; Piri, M. Heat of capillary condensation in nanopores: new insights from the equation of state. Phys. Chem. Chem. Phys., 2017, 19, 5540-5549.

15. Gor, G. Y.; Paris, O.; Prass, J.; Russo, P. A.; Ribeiro Carrott, M. M. L.; Neimark, A. V. Adsorption of n-pentane on mesoporous silica and adsorbent deformation. Langmuir, 2013, 29, 8601-8608. 